\title{
CHARACTERIZATIONS \\ OF \\ INFORMATION \\ MEASURES
}


This page is intentionally left blank 


\title{
CHARACTERIZATIONS OF
}

\author{
INFORMATION
} MEASURES

\author{
Bruce Ebanks \\ Department of Mathematics, Marshall University, \\ Huntington, WV 25755, USA

\section{Prasanna Sahoo} \\ Department of Mathematics, University of Louisville, \\ KY 40292, USA \\ Wolfgang Sander \\ Institute for Analysis, Technical University, Pockelsstrasse \\ 14, D-38106, Braunschweig, Germany
}




\section{Published by}

World Scientific Publishing Co. Pte. Ltd.

P O Box 128, Farrer Road, Singapore 912805

USA office: Suite 1B, 1060 Main Street, River Edge, NJ 07661

UK office: 57 Shelton Street, Covent Garden, London WC2H 9HE

\section{British Library Cataloguing-in-Publication Data}

A catalogue record for this book is available from the British Library.

\section{CHARACTERIZATIONS OF INFORMATION MEASURES}

Copyright $\odot 1998$ by World Scientific Publishing Co. Pte. Ltd.

All rights reserved. This book, or parts thereof, may not be reproduced in any form or by any means, electronic or mechanical, including photocopying, recording or any information storage and retrieval system now known or to be invented, without written permission from the Publisher.

For photocopying of material in this volume, please pay a copying fee through the Copyright Clearance Center, Inc., 222 Rosewood Drive, Danvers, MA 01923, USA. In this case permission to photocopy is not required from the publisher.

ISBN $981-02-3006-0$

Printed in Singapore. 


\author{
Dedicated by \\ Bruce Ebanks \\ to his wife Annalisa \\ and \\ his teacher Che Tat Ng, \\ and \\ Prasanna Sahoo \\ in memory of his father Hari \\ and \\ to his teacher Pallaniappan Kannappan, \\ and \\ Wolfgang Sander \\ to his wife Helda \\ and \\ daughters Michelle and Christin
}


This page is intentionally left blank 


\section{PREFACE}

This book is a monograph on the characterizations of various measures of information. It has arisen from over fifteen years' research on the subject, much of which has been done by the authors. In this book we treat various properties of measures of information and the extent to which these properties characterize known measures of information. To the best of our knowledge this is the first book investigating this topic on open domains in depth; an earlier book by Aczél and Daróczy (1975) treated the subject on closed domains.

The book is written for students, educators, and practitioners of information theory, statistics, and functional equations. The goal of the book is twofold. The first goal is to provide the interested reader with up-to-date information about the characterization of information measures on open domains. The second is to present a comprehensive illustration of the use of functional equations to model and solve problems in information theory. Not many prerequisites are needed to understand this book. Some basic calculus, measure, probability and set theory facts, which can be found in almost all textbooks on these subjects, are sufficient.

We rely heavily upon the theory of functional equations, but we have presented in this book essentially everything that is needed in that regard. That is, the book includes all results about functional equations that we use in the characterizations of information measures. Indeed this book can be treated as a book on functional equations with applications to information theory. Additional information can be found in several books on functional equations, such as Aczél (1966), Kuczma (1985) or Aczél and Dhombres (1989). Another interesting feature is a detailed coverage of sum form information measures in a very general setting.

The book contains nine chapters. Chapter 1 includes some basic results from the theory of functional equations. In Chapter 2, we treat the branching property of information measures and discuss the characteristic functional equation for these measures. This chapter concludes with the symmetric solution of the cocycle equation. Chapter 3 presents some results regarding recursivity properties of information measures. Here, we treat recursivity in a very general form, the recursivity of multiplicative type. Chapter 4 deals with 
the fundamental equation of information and presents the forms of recursive measures which satisfy some regularity property such as measurability.

The last five chapters cover the characterizations of sum form information measures which arise from various types of additivities. Chapter 5 presents, in addition to the classical additivity property of Shannon, two other more general types of additivity, known as additivity of type $\lambda$ and additivity of type $\left(M_{1}, M_{2}\right)$. In this chapter the reader will also find a justification for the study of information measures with these additivity properties. Chapter 6 covers some basic sum form functional equations. These equations are instrumental in characterizations of all additive sum form information measures of type $\lambda$ as well as all additive sum form information measures of type $\left(M_{1}, M_{2}\right)$. All additive sum form information measures with measurable generating function are explicitly determined in Chapter 7. Such measures are shown to be a linear combination of Kerridge's inaccuracy, entropies of degree one and zero, and a constant. In Chapters 8 and 9, the forms of all additive sum form information measures of type $\lambda$ and of all weighted additive sum form information measures of type $\left(M_{1}, M_{2}\right)$ are determined, respectively.

We do not claim that everything written in this book originated with us. In fact, we freely use the works of many researchers, and we have attempted to acknowledge all contributions. For some results we present new proofs which do not appear elsewhere. In other cases, we did not succeed in improving on the original explanations. Nonetheless we feel that it is worthwhile to have all these results gathered in one place and linked together in a coherent fashion. We hope the readers will agree.

Donald Knuth's TEXprogram was used to create the manuscript. We wish to thank Betty Hampton for her valuable assistance in typing a large part of the manuscript.

Bruce Ebanks, Huntington

Prasanna Sahoo, Louisville

Wolfgang Sander, Braunschweig 


\section{CONTENTS}

1. Introduction, Preliminaries and Notation . . . . . . . . . 1

1.1. Introduction and notation . . . . . . . . . . . . . . . 1

1.2. Functional equation preliminaries . . . . . . . . . . . . 6

1.3. A general regularity theorem . . . . . . . . . . . . . . 21

2. The Branching Property . . . . . . . . . . . . . . . . . . 29

2.1. Introduction . . . . . . . . . . . . . . . . . . . . . 29

2.2. The characteristic functional equation for branching measures of information . . . . . . . . . . . . . . . 31

2.3. Symmetric branching information measures . . . . . . . . 41

2.4. Partially symmetric branching information measures . . . . 44

2.5. Historical remarks . . . . . . . . . . . . . . . . . . . 48

3. Recursivity Properties . . . . . . . . . . . . . . . . . . 51

3.1. Introduction . . . . . . . . . . . . . . . . . . . 51

3.2. Recursivity of multiplicative type . . . . . . . . . . . . 53

3.3. Nonadditive multiplicative types . . . . . . . . . . . . 57

3.4. Additive multiplicative types . . . . . . . . . . . . . . 60

3.5. Summary and further remarks . . . . . . . . . . . . . 77

4. The Fundamental Equation of Information and Regular Recursive Measures . . . . . . . . . . . . . 81

4.1. Introduction . . . . . . . . . . . . . . . . . . . . . 81

4.2. General solution of the fundamental equation of information . . . . . . . . . . . . . . . . . . . . 84

4.3. Regular recursive measures . . . . . . . . . . . . . . . 88

4.4. Summary . . . . . . . . . . . . . . . . . . . . . 102

5. Sum Form Information Measures

and Additivity Properties . . . . . . . . . . . . . . . . 107

5.1. Introduction . . . . . . . . . . . . . . . . 107

5.2. Additivity . . . . . . . . . . . . . . . . . . . . 107

5.3. Generalized additivities . . . . . . . . . . . . 108

5.4. Weighted additivity . . . . . . . . . . . . . 113 
6. Basic Sum Form Functional Equations . . . . . . . . . . 117

6.1. Introduction . . . . . . . . . . . . . . . . . . . . 117

6.2. Measurable solutions of (6.1.1) in the 1-dimensional case . 118

6.3. Measurable solutions of (6.1.1) in higher dimensions . . . 130

6.4. Some generalized Cauchy equations . . . . . . . . . . 137

6.5. Some linear independence results on additive and multiplicative functions . . . . . . . . . . . . . . 149

7. Additive Sum Form Information Measures . . . . . . . . 157

7.1. Introduction . . . . . . . . . . . . . . . . . . . . 157

7.2. Solution of (5.2.2) in the case $m \geq 3$ or $\ell \geq 3$. . . . . . 158

7.3. Solution of (5.2.2) in the case $\ell=2=m$. . . . . . . . 162

7.4. Some heuristic arguments . . . . . . . . . . . . 166

8. Additive Sum Form Information Measures of Type $\lambda$. . . . . . . . . . . . . . . . . . . . . . . . . . . . . . . . . . 171

8.1. Introduction . . . . . . . . . . . . . . . . . . . . 171

8.2. A general structure theorem . . . . . . . . . . . . . 174

8.3. Solution of (5.3.12) in the case $m \geq 3$ or $\ell \geq 3$. . . . . 188

8.4. Solution of (5.3.12) in the case $\ell=m=2$. . . . . . . 201

9. Additive Sum Form Information Measures of Multiplicative Type . . . . . . . . . . . . . . . . . . 221

9.1. Introduction . . . . . . . . . . . . . . . . . . . . 221

9.2. Preliminaries . . . . . . . . . . . . . . . . . . . . 222

9.3. Solution of (5.4.7) in the case $m \geq 3$ or $\ell \geq 3$. . . . . . 234

9.4. Solution of (5.4.7) in the case $\ell=m=2$. . . . . . . . 239

9.5. Summary . . . . . . . . . . . . . . . . . . . . . 258

References . . . . . . . . . . . . . . . . 261

Index . . . . . . . . . . . . . . . . . . . . . . . 277 